\title{
AN INEQUALITY FOR COMPLEX LINEAR GROUPS OF SMALL DEGREE
}

\author{
HARVEY I. BLAU
}

ABSTRACT. Let $G$ be a finite complex irreducible linear group of degree less than $p-1$ for some fixed prime $p$, whose order is divisible by $p$ to the first power only, and which has no normal Sylow $p$-subgroup. An inequality of Brauer, which bounds $p$ by a function of the number of conjugate classes of $p$-elements, is improved.

The purpose of this note is to prove

THEOREM 1. Let $G$ be a finite group with the following properties: for some fixed prime $p$, a Sylow $p$-subgroup $P$ of $G$ has order $p$ and is not normal in $G$; the number $t$ of conjugate classes of p-elements of $G$ is at least 3 ; and $G$ has a faithful irreducible complex character $\chi$ of degree $d<p-1$. Then $p \leqq t^{2}-3 t+1$.

This improves Brauer's inequality $p \leqq t^{3}-t+1$ [2]. Hayden's result [6] that $t \geqq 6$ follows from Theorem 1 after the case $t=5, p=11$ is handled. All groups with $t \leqq 2$ and which satisfy the other hypotheses of Theorem 1 are known [8]. Apparently, no groups are known which satisfy these hypotheses with $6 \leqq t<(p-1) / 2$.

The proof is basically a series of observations on the methods of [5]. Note that [5, Theorem 1] shows that either $t$ is even or $t=(p-1) / 2$.

Proof. Assume the hypotheses of Theorem 1. Then $d=p-(p-1) / t$ [2]. If $p=7$, no such groups exist [3], so we may assume $p>7$.

Let $e=(p-1) / t$. If $e \leqq 2$, then $t \geqq(p-1) / 2$, and the theorem follows trivially for $p>7$. Assume henceforth $e>2$.

Let $G_{1}$ be the normal closure of $P$ in $G$, and let $G_{2}=G_{1}^{\prime}$. Feit's reduction argument $[5,(6.1)]$ shows that $G_{2}=G_{2}^{\prime}, G_{2} / Z\left(G_{2}\right)$ is simple, and $G_{2} / \mathcal{Z}\left(G_{2}\right) \nsim P S L_{2}(p)$. Also, $G_{2}$ and $\left.\chi\right|_{G_{2}}$ satisfy the hypotheses of Theorem 1. Since $d$, and hence $t$, is the same for both groups, it suffices to prove the theorem in case $G=G_{2}$. Then by $[5,(2.1)], G$ satisfies conditions $\left(^{*}\right)$ of $[5]$ and $|\mathrm{Z}(G)| \mid p-e$. Thus [5, Theorem 1] implies $e$ is odd and $t$ is even.

For the rest of this paper we use the following notation, in accordance with [5]: $N=\Re_{G}(P) ; Z=Z(G) ; B_{u}$ is a $p$-block of defect 1

Received by the editors May 25, 1970.

AMS 1969 subject classifications. Primary 2025, 2080; Secondary 2075.

Key words and phrases. Faithful irreducible complex representation, complex character, prime order Sylow subgroup, small degree, conjugate class of $p$-elements.

Copyright @ 1971, American Mathematical Society 
corresponding to a linear character $\eta^{u}$ of $Z$. The exceptional characters in $B_{u}$ are denoted by $\chi_{j}^{(u)}, 1 \leqq j \leqq t$. The sign $\delta_{u}= \pm 1$ as $\chi_{j}(1)$ $\equiv \pm e(\bmod p) . \mathcal{O}$ is the ring of integers in a $p$-adic number field, $\beta$ is the maximal ideal of $\theta$, and $K=\mathcal{\theta} / \odot$. If $X$ is an $\theta G$-module, $\bar{X}$ $=X / \odot X$. A typical indecomposable $K N$-module is denoted $V_{r, u}^{\lambda}$ where $1 \leqq r \leqq p$ and $1 \leqq u \leqq|Z| . \lambda=\alpha^{h}$ for some integer $h$, where $\alpha$ is the linear character of $N$ given by $g^{-1} y g=y^{\alpha(g)}$, all $g \in N, y \in P$.

The following lemma is an obvious modification of $[\mathbf{5},(3.8)]$. It is proved by making the corresponding obvious changes in the proof of that result.

LEMмA 2. Let group $J$ satisfy $\left(^{*}\right)$ with $t \geqq 3$, and let $M$ be an 0 -free OJ-module which affords the character $\xi$. Suppose that $\left.\xi\right|_{z}=\xi(1) \eta^{u}$ where $\delta_{u}=1$. Let $\xi=\alpha+\beta+\gamma$ where $\alpha=\sum_{j=1}^{t} h_{j} \chi_{j}^{(u)}, \beta$ is a character in $B_{u}$ which is orthogonal to every $\chi_{j}^{(u)}$, and $\gamma$ is orthogonal to every character in $B_{u}$. Let $h=\sum_{j=1}^{t} h_{j}$. Then the following hold:

(i) If $\bar{M}$ is indecomposable and $\xi(1) \equiv e(\bmod p)$ or $\xi(1) \equiv e+1$ $(\bmod p)$ then $h \geqq 1$.

(ii) If $\bar{M}=W_{1} \oplus W_{2}$ where each $W_{i}$ is indecomposable and $\xi(1)$ $\equiv 2 e(\bmod p)$ then $h \geqq 2$.

Now let $\chi$, as in the statement of Theorem 1 , be in the $p$-block $B_{u} . \chi$ is an exceptional character $[5, \S 2]$. Let $X$ be an $\theta$-free $\theta G$ module which affords $\chi$ such that $\bar{X}$ is indecomposable. Then $\left.\bar{X}\right|_{N}$ $=V_{p-e, u}^{\mu}$ for some $\mu \in\langle\alpha\rangle$, and by [4, Lemma 3.7],

$$
\left.(\bar{X} \otimes \bar{X})\right|_{N}=\oplus \sum_{k=0}^{e-1} V_{2 k+1, u+u}^{\mu^{2} \alpha^{k}} \oplus \sum_{k=e}^{p-e-1} V_{p, u+u}^{\mu^{2} \alpha^{k}} .
$$

Since $P$ is a T.I. set, for each $0 \leqq k \leqq e-1$ there is a unique indecomposable $K G$-module $W_{k}$ so that $V_{2 k+1, u+u}^{\mu^{2} \alpha^{k}}$ is the unique nonprojective indecomposable summand of $\left.W_{k}\right|_{N}$. Then

$$
\bar{X} \otimes \bar{X}=\oplus \sum_{k=0}^{e-1} W_{k} \oplus S,
$$

where $S$ is projective. There is a subset $S_{k}$ of the integers $j$ with $e \leqq j \leqq p-e-1$ such that, from (3),

$$
\left.W_{k}\right|_{N}=V_{2 k+1, u+u}^{\mu^{2} \alpha^{k}} \oplus \sum_{j \in \mathcal{S}_{k}} V_{p, u+u}^{\mu^{2} \alpha^{j}}
$$

Assume $\theta$ is large enough so that, by [5, (3.6)], for each $k$ with $0 \leqq k<e$, there is an $\theta$-free $\theta G$-module $M_{k}$ such that $\bar{M}_{k} \approx W_{k} \oplus W_{e-k-1}$, and so that there exists an $\theta$-free $\theta G$-module $L$ with $\bar{L} \approx W_{(e-1) / 2}$. 
Let $\zeta$ be an exceptional character in $B_{u+u}$. By $[5,(4.1)], \delta_{u+u}=1$, so that $\zeta(1) \equiv e(\bmod p)$. Since $\zeta(1) \neq p-e$, we have $\zeta(1)>p$. Let $Y$ be a modular constituent of $\zeta$. Let $\operatorname{dim}_{K} Y=a p+y, 0<y<p$. By an argument of Rothschild [7], $\sum y=e$, where the sum is taken over all modular constituents of $\zeta$. It follows that there exists some constituent $Y$ with $\operatorname{dim}_{K} Y>p$. Then $\left.Y\right|_{N}$ contains at least one projective summand $V_{p, u+u}^{\gamma}$.

By Lemma 2, for each $0 \leqq k<e$, the $p$-conjugates of $\zeta$ occur in the character afforded by $M_{k}$ with a total multiplicity of at least 2 . Thus $Y$ occurs with multiplicity at least 2 as a constituent of $W_{k} \oplus W_{e-k-1}$. Similarly, $Y$ occurs with multiplicity at least 1 as a constituent of $W_{(e-1) / 2}$. Thus $V_{p, u+u}^{\gamma}$ occurs at least twice in a direct sum decomposition of $\left.\left(W_{k} \oplus W_{e-k-1}\right)\right|_{N}$, and at least once in a decomposition of $\left.W_{(e-1) / 2}\right|_{N}$. Hence $V_{p, u+u}^{\gamma}$ occurs at least $2(e-1) / 2+1=e$ times in the decomposition (3). Since $|\langle\alpha\rangle|=e, V_{p, u+u}^{\gamma}$ can occur at most $t-1$ times, and at most $t-2$ times unless $\gamma=\mu^{2} \alpha^{e}=\mu^{2}$.

Suppose $\gamma=\mu^{2}$ and $e=t-1 . \bar{X} \otimes \bar{X}$ is the direct sum of symmetric and skew summands, and by [1, Lemma 3.3], $W_{k}$ and $W_{e-k-1}$ are both symmetric summands for $k$ odd, and both skew summands for $k$ even, since $e$ is odd. $V_{p, u+u}^{\gamma}$ occurs exactly twice as a summand of each $\left.\left(W_{k} \oplus W_{e-k-1}\right)\right|_{N}$, and exactly once in $\left.W_{(e-1) / 2}\right|_{N}$. It follows that $V_{p, u+u}^{\gamma}$ is a skew summand of $\left.(\bar{X} \otimes \bar{X})\right|_{N}$ more times than it is a symmetric summand. However, [1, Lemma 3.3] also shows that $V_{p, u+u}^{\mu^{2}}$ appears $t / 2$ times as a symmetric summand and $(t / 2)-1$ times as a skew summand: contradiction.

Thus $e \leqq t-2$. Since $e$ is odd and $t$ even, we have $e \leqq t-3$. Since $e=(p-1) / t$, it follows that $p \leqq t^{2}-3 t+1$, and Theorem 1 is proved.

Corollary 4. Assume the hypotheses of Theorem 1. Then

$$
d \geqq p+(3 / 2)-(p+5 / 4)^{1 / 2} .
$$

Proof. We know $e \leqq(p-1) / e-3$, so $e^{2}+3 e-(p-1) \leqq 0$. Hence $e \leqq-3 / 2+(p+5 / 4)^{1 / 2}$. Since $d=p-e$, we are done.

\section{REFERENCES}

1. H. I. Blau, Under the degree of some finite linear groups, Northern Illinois University, DeKalb, Ill., 1970 (preprint).

2. R. Brauer, Some results on finite groups whose order contains a prime to the first power, Nagoya Math. J. 27 (1966), 381-399. MR 33 \#7402.

3. - Über endliche lineare Gruppen von Primzahlgrad, Math. Ann. 169 (1967), 73-96. MR 34 \#5913.

4. W. Feit, Groups with a cyclic Sylow subgroup, Nagoya Math. J. 27 (1966), 571-584. MR $33 \# 7404$. 
5. - - On finite linear groups, J. Algebra 5 (1967), 378-400. MR 34 \#7632.

6. S. Hayden, On finite linear groups whose order contains a prime larger than the degree, Thesis, Harvard University, Cambridge, Mass., 1963.

7. B. Rothschild, Degrees of irreducible modular characters of blocks with cyclic defect groups, Bull. Amer. Math. Soc. 73 (1967), 102-104. MR 34 \#4381.

8. H. F. Tuan, On groups whose orders contain a prime number to the first power, Ann. of Math. (2) 45 (1944), 110-140. MR 5, 143.

Northern Illinois University, DeKalb, Illinois 60115 